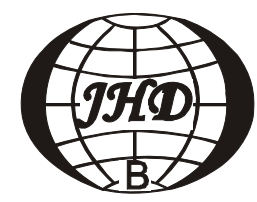

sdlj.chinajournal.net.cn

\title{
THE INVESTIGATION OF VORTEX METHOD AND ITS APPLICATION TO DRAG REDUCTION TECHNOLOGY
}

\author{
Ph.D.Candidate: GAO Li-jin \\ China Ship Scientific Research Center, Wuxi 214082, China, E-mail: glj2001@sina.com
}

\author{
Supervisor: \\ Zhou Lian-di (CSSRC) \\ Zhu De-xiang (CSSRC) \\ Members of Dissertation Defense Committee: \\ Chairman \\ Miao Guo-ping (Shanghai Jiaotong University), \\ Dai Shi-qiang (Shanghai University) \\ Dong Shi-tang (CSSRC) \\ Li Bai-qi (CSSRC) \\ Sheng Hong-cui (CSSRC) \\ Tao Ming-de (Fudan University) \\ Zhou Lian-di (CSSRC)
}

Time of Dissertation Defense: Apr.7, 2006

\begin{abstract}
The flow past a circular cylinder and airfoil with varying mathematical roughness function are numerically simulated. A new model about blowing and suction is constructed by using the concept of mathematical roughness function. The flow field and the drag are investigated through this new model. By the numerical study about bluff body, some conclusions are drawn to reduce the drag.
\end{abstract}

KEY WORDS: mathematical roughness function, sublayer of laminar flow, blowing and suction mechanism, drag reduction respond time

BRIEF INTRODUCTION OF THE PAPER: In this paper, the vortex method, the new concept mathematical roughness introduced from the boundary condition of vortex method and the new model based on the mathematical roughness function are presented. The flow around two and three dimensional bluff bodies was investigated. The new research method about turbulence drag reduction was given in this paper.

The numerical simulation about the circular cylinder started impulsively was studied. The vorticity contours, streamlines and drag coefficient curve were gotten. The comparison between the results of this paper and the computational results from published paper was made and agreed. So the computational method of this dissertation is reliable.

The circular cylinder and airfoil with uniform distributed mathematical roughness were numerical simulated. Some important conclusions were drawn. If minus mathematical roughness was enforced uniformly, the thrust would be generated. If the mathematical roughness between 0 and 1 was enforced uniformly, the drag would be reduced. If the mathematical roughness grater than 1 was enforced uniformly, the drag would be increased. The drag of the cylinder with mathematical roughness equal to 1.5 showed a very interesting phenomenon. At the beginning the drag of mathematical roughness surface was greater than the drag of normal surface, but after a period these two drags became same. This phenomenon agreed with the traditional roughness concept. This conclusion gave a proof to the interpretation of the mathematical roughness.

The cylinder with blowing and suction was simulated by using the new blowing and suction model based on the mathematical roughness function. Some conclusions were drawn. Among them, the conclusion that blowing at the shoulder of cylinder could reduce the drag agreed with the conclusions of published papers. So the correction of the new model was proved.

Lastly, the flow around a three underwater aircraft was computed, the vorticity contours were gotten. So it could be said that the computational method in this dissertation was feasible.

In general, this dissertation provides practical and feasible research method to investigate two and three dimensional unsteady flow and turbulence drag reduction. 